\title{
Multi-messengers at ultra-high energies with the Pierre Auger Observatory
}

\author{
Julien Souchard $^{1, *}$, for the Pierre Auger Collaboration ${ }^{2, a}$ \\ ${ }^{1}$ Laboratoire de Physique Subatomique \& Cosmologie, 38026 Grenoble, France \\ ${ }^{2}$ Observatorio Pierre Auger, Av. San Martin Norte 304, 5613 Malargüe, Argentine
}

Received 18 December 2019, Accepted 4 May 2020

\begin{abstract}
The Pierre Auger Observatory is an Ultra-High Energy Cosmic Ray (UHECR) detector which has studied cosmic particles with energies above and around $10^{18} \mathrm{eV}$ for more than 15 years. It has proved to be the most competitive instrument at these energies and has produced a wealth of valuable results, improving our understanding of UHECRs. A complete understanding of these highest energy particles is crucial to understand the extreme astrophysical events in which they are produced and accelerated, as well as their propagation to Earth. In the same range of energies, UHE photons and neutrinos are of paramount importance as, being electrically neutral, they point back to their origin while charged particles are deflected in the galactic and extragalactic magnetic fields. The flux of extragalactic photons, neutrinos, and cosmic rays are believed to be highly linked, by their origin and their interactions. Each messenger provides different information about the potential sources, and having detection means for all four messengers, including gravitational waves, allows us to shed light on energetic sources of astroparticles. The Pierre Auger Observatory benefits from a large exposure and a good angular resolution, and is efficient in detecting UHE photons and neutrinos. These performances make possible follow-up searches for events detected by gravitational waves, such as the binary mergers observed by the LIGO/Virgo detectors, or any other energetic sources of particles.
\end{abstract}

Keywords: Multi-messenger, Pierre Auger observatory, UHECR, Gravitational waves, Neutral particles, Binary black hole mergers, UHE photons, UHE neutrinos

\section{Introduction}

Ultra-High Energies Cosmic Rays (UHECRs) are ionized atomic nuclei that travel through the universe and are detected on Earth at energies above $10^{18} \mathrm{eV}$, up to few times $10^{20} \mathrm{eV}[1,2]$. The CR spectrum, spanning an energy range from $10^{13} \mathrm{eV}$ to $10^{21} \mathrm{eV}$ is presented in Figure 1. This spectrum is made by using the data of various experiments, each measuring a different range of energy, based on their detection settings. The spectrum follows a power law with a spectral index around $\gamma=-3\left(E^{\gamma}\right)$ but variations of this index are observed in few particular points. At UHE, they arrive at the top of Earth's atmosphere in a very limited flux, less than 1 particle per square kilometer per year [2], making their detection challenging. But at these energies, the cosmic rays interacting with the atmosphere generate Extensive Air Showers (EAS) [3, 4]. When an UHECR primary interacts with a nucleus in the upper atmosphere, it initiate a cascading process resulting in the creation of a "shower" of relativistic

\footnotetext{
*Corresponding author: souchard@lpsc.in2p3.fr

${ }^{a}$ Full author list: http://www.auger.org/archive/authors_2019 07.html
}

particles that travels through the atmosphere some of which reach the ground. To give an order of magnitude, a shower initiated by a $10^{19} \mathrm{eV}$ proton is made up of about $5 \times 10^{10}$ particles (mostly $e^{ \pm}, \mu^{ \pm}, \gamma$ ) at ground-level [5, 6].

To study UHECRs, observatories [7-11] looking at EAS have been built, like the Pierre Auger Observatory. It has now been shown that combining UHECRs observations with other messengers to study astrophysical events has a lot of potential and that multimessenger astronomy has great prospects of making discoveries in the future.

Although galactic cosmic rays are sometimes interpreted as being Fermi accelerated in shocks around supernova [12], the origin of extragalactic cosmic rays remains a mystery [13]. The acceleration mechanisms that generate UHECRs are still not fully understood, we know however that some minimum requirements have to be met. This "Hillas condition" [14] states that an accelerator must have a radius $R>r_{\text {Lar }}$ in order to be able to magnetically confine a particle with gyroradius $r_{\text {Lar }}$. Ultra-high-energy sources should be able to confine cosmic rays within a sufficiently magnetized and large region to accelerate them up to a rigidity $R=E / Z$, where $E$ is the energy and $Z$ the charge of each nucleus. 


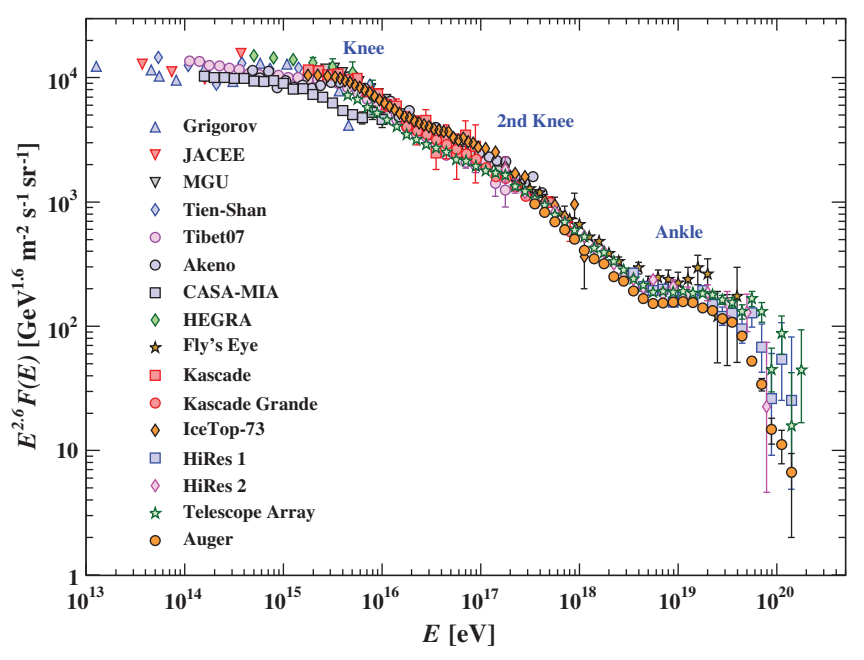

Figure 1. Charged cosmic ray spectrum measured from $10^{13} \mathrm{eV}$ to few $10^{20} \mathrm{eV}$ by cosmic ray observatories. The spectrum features corresponding to inflections points around $10^{16} \mathrm{eV}, 10^{17}$ $\mathrm{eV}$, and $10^{19} \mathrm{eV}$ are highlighted with the "knee", "2nd knee", and the "ankle". UHECRs are in the range of energies above $10^{18} \mathrm{eV}$. Taken from [44], see also [45] and references therein.

The available energy and timescale of the acceleration is also a factor, as well as the energy loss processes within the acceleration site. Just accelerating a single particle to energies up to $10^{20} \mathrm{eV}$ is challenging, but a plausible source candidate must also satisfy the energy budget constrained by UHECRs observations. Several objects are considered as UHECRs source candidates: GRBs and energetic supernova, AGNs, tidal disruptions events, starbusts galaxies, galaxy clusters, and pulsars (an overview of these potential candidates can be found in [15]). Binary black holes merger could be a potential acceleration site of extragalactic CRs. Long-lived debris orbiting around the mergers could be injected at the time of the merging and be accelerated to ultra-high energies [16].

Other UHECRs generation processes have been proposed, like the top-down process, in which UHECRs are created by the decay of super heavy particles, but these models have mostly been excluded by observational data [17]. In the rest of this article, neutral particles (neutrinos and photons) and hadronic primaries (protons and heavier charged nuclei) will be discussed seperately. This separation is motivated on one part by the differences in their attributes and also because the detection of neutral particles with the Pierre Auger Observatory requires specific analyses.

Also, the neutral primaries are probes into specific astrophysics scenarios. UHE neutrinos and photons can be produced at the sources or during the UHECR propagation (see Sect. 3) and they could provide clues to undertstand whether the end of the cosmic-ray spectrum (the sharp drop in flux oberved beyond $\sim 10^{20}$ ) is due to the GZK effect or to a maximum rigidity scenario, where the sources simply reach their maximum acceleration power.

It is becoming more and more crucial to perform multi-messenger observations of a single astrophysical phenomenon. By combining the different types of messengers, photons (at multiple wavelengths), cosmic rays, neutrinos, and gravitational waves, we can learn more about our universe and better constrain our astrophysical models. Particle acceleration around compact objects is not very well understood at the moment, and studying merging processes, with informations on the objects (mass, energy radiated as GW, collapse time, etc.) would help us establish new, more refined acceleration models to the highest energies $[16,18]$. There is an interplay between the different kinds of particles. For example, photons can interact with cosmic rays and cosmic rays can emit photons and neutrinos when they decay. Another interest in combining the different messengers, is the complementarity between the observations. Let us list some of the pros and cons of each messenger:

- Charged cosmic rays:

- Pros: they are the only direct accelerator probe, as they are the particles that are actually being accelerated in the sources.

- Cons: they are deflected in magnetic fields. This means that their arrival directions are smeared, but also that they are delayed on their way to Earth.

- Gamma-rays:

- Pros: as they are not charged, they propagate from their sources to Earth in a straight line. Also, photons are historically a privileged messenger for astrophysics and are thus very well known in this context.

- Cons: since they interact with matter easily, especially at ultra-high energy, the horizon for UHE photons is less than $10 \mathrm{Mpc}$.

- Neutrinos:

- Pros: like photons, neutrinos travel through the universe unperturbered by magnetic fields. Additionally they interact very rarely with any kind of matter, so unlike UHE photons, their horizons is not an issue. With almost no horizon,there is a vast number of potential sources.

- Cons: since they interact rarely with matter, this makes their detection difficult (need high exposure). With potentially almost no horizon for neutrinos, ${ }^{1}$ one could expect an isotropic diffuse background of neutrinos.

With the advent of gravitational wave detection with ground-based interferometers, marked by the successful observation of binary mergers by the LIGO/Virgo collaboration (a few relevant events are discussed later), alerts can be sent to multiple observatories, allowing for multimessenger studies of transient events. The Pierre Auger Observatory can take part in these multi-messenger observations, by searching for signals in time-windows close to the astrophysical events. Of particular interest are photons and neutrinos, neutral particles, whose arrival direction and time of emission can be inferred with better precision. The Pierre Auger Observatory's role in multi-messenger studies is crucial to help understanding astrophysical phenomena, up to the highest energies.

\footnotetext{
${ }^{1}$ Universe expansion/propagation speed limits.
} 


\section{Observing ultra-high energy particles with the Pierre Auger Observatory}

The Pierre Auger Observatory [19] has been studying cosmic rays for around 15 years. It is located in the Argentinian pampa, in the province of Mendoza, near the town of Malargüe. It is exploited by an international collaboration of about 400 scientists from 18 countries. It mainly consists of the association of two types of detectors: a surface detector and a fluorescence detector.

The surface detector (SD) is an array of 1660 Water-Cherenkov Detectors (WCD), spread throughout $3000 \mathrm{~km}^{2}$ in a triangular pattern, with a spacing between two detectors of $1.5 \mathrm{~km}$. A denser array, with a spacing of $750 \mathrm{~m}$, is nested inside the SD, it spans a surface area of $23.5 \mathrm{~km}^{2}$. It has been designed to study less energetic EAS down to $10^{17} \mathrm{eV}$. Several scintillator detectors are buried close to a few WCD to study the muon content of the showers. Each WCD measures the timing and signal of the secondary particles going through it, taking a snapshot of the developing shower as it hits the ground. This information is then used to reconstruct the showers' development, in particular its energy and arrival direction, and to infer its nature. The SD has a duty cycle of about $\sim 100 \%$.

The fluorescence detector (FD) consists of 27 telescopes, located in four sites, overlooking the surface array. Of those 27 , three telescopes can be tilted upward $29^{\circ}$, in an effort to look at nearby low-energy showers in correlation with the denser array, which is centered about $6 \mathrm{~km}$ away from them. These telescopes record the fluorescence light emitted by the excited nitrogen atoms in the atmosphere along the showers' development path. The FD performs a calorimetric measurement of the shower's energy and its depth at maximum. A drawback is that it can only operate on clear moonless nights, so it has a duty-cycle of about $\sim 13 \%$.

The hybrid detection (SD + FD) is more than just the sum of the two detections. The SD only has an indirect energy measurement, so the value measured with the FD is used to calibrate it. Figure 2 shows an exceptionnal event, where the high energy and central position of the EAS, allowed it to be detected by fluorescence telescopes in all four FD sites, as well as the SD at the same time. With hybrid events $(\mathrm{FD}+\mathrm{SD})$, we get a high-precision dataset of hybrid events, extremely useful for several analyses. Other types of measurements are done on the EAS at the Pierre Auger Observatory, but on a smaller scale. A sub array is equipped with radio antennas [20], others have nearby underground muon counters [21]. As it will be discussed later, scintillator detectors and radio antennas will be deployed on a bigger scale (almost the whole surface array). With the Pierre Auger Observatory, a lot of ground has been unraveled in cosmic ray physics, be it on the UHECRs arrival direction, their spectrum and composition. In this work we will focus on multimessenger astrophysics and neutral particles (photons and neutrinos).

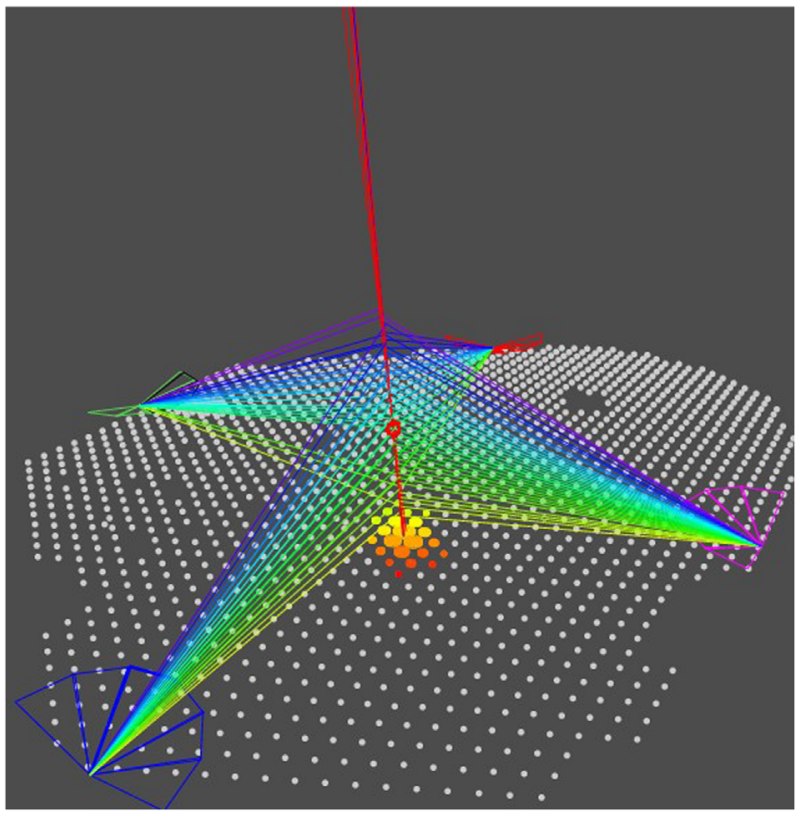

Figure 2. Representation of an event reconstructed by the Pierre Auger Observatory hybrid detection technique. The white dots corresponds to the WCD making up the SD, with the colored ones being the WCD activated by the shower. The four polygons around the SD are the buildings where the fluorescence telescopes are located and the straight red line is the axis of an EAS hitting the array.

\section{Neutral particles searches}

The Pierre Auger Observatory has proven that aside from its main target, the nucleus primaries, it also has good sensitivity to photon and neutrino primaries. From the limits set by the Pierre Auger Observatory on UHE photon and neutrino fluxes, most top-down UHECR generation processes have already been rejected, and other astrophysical scenarios can be explored. Dedicated analyses have been designed to look specifically for these kind of primaries, using the development of the shower to distinguish them from hadronic primaries or exploring channels where the background is almost non-existent.

\section{Photon searches}

The search for UHE photons is very important to study transient and steady sources as well as their environment and also to constrain some astrophysical scenarios. As an example, the limits set by the Pierre Auger Observatory on the UHE photon flux [22] have been used to reject some top-down UHECRs production models [17, 23] and can be used to constrain potential Lorentz invariance violation processes $[24,25]$.

An even more obvious scenario to study in the context of UHE photons is the Greisen-Zatsepin-Kuzmin (GZK) suppression [26, 27]. The GZK effect is the expected strong suppression in the flux of UHECRs around $10^{20} \mathrm{eV}$ caused 
by the interaction (1) of UHECRs with the Cosmological Microwave Background (CMB) photons:

$$
\begin{aligned}
& p+\gamma_{\mathrm{CMB}} \rightarrow \Delta^{+}(1232) \rightarrow p+\pi^{0} \\
& p+\gamma_{\mathrm{CMB}} \rightarrow \Delta^{+}(1232) \rightarrow n+\pi^{+}
\end{aligned} .
$$

The flux suppression at UHE is undisputably confirmed by the data from the Pierre Auger Observatory, however it has not yet been proven whether this comes from the GZK effect or if this is merely the maximum energy at the sources in a maximum rigidity scenario [28]. Finding an excess of UHE photons at energies immediately below a hypothetical GZK-energy threshold for protons, could be a proof of the GZK effect.

To search for those potential photons in the data of the observatory, one must look at the features of the EAS and extract useful observables. In general, these discriminant observables are constructed by comparing photon-induced to proton-induced showers because they are the most similar (heavier primaries will reinforce the differences). Two phenomena/EAS features used are schematized in Figure 3:

- In photon-initiated showers, the maximum of shower development (the depth at which the number of particles in the shower is maximum) is deeper in the atmosphere.

- There are fewer muons in photon-induced EAS.

Both phenomena have the same root cause: there are less hadrons in a photon-initiated shower. EAS have an electromagnetic (EM) and a hadronic components and most of the primary's energy is spread out in the first few interactions. In a photon-induced shower, the first few interactions do not produce as many hadrons $[29,30]$ so less energy is available for the hadronic component of the shower. The hadronic component of a shower develops faster than the EM component since hadronic interactions produce more secondary particles and thus spread out the energy. As a consequence, the photon-induced showers develop deeper into the atmosphere than one from a hadronic primary. Similarly, since more muons are produced in hadronic interactions than electromagnetic ones, the smaller hadronic component explains why there are less muons on the ground for photon-induced showers. So far, no UHE photons have been detected, but the Pierre Auger Observatory has proven its good sensitivity to photon primaries [31], it is thus able to derive good constraints on the diffuse photon flux from $3 \times 10^{17} \mathrm{eV}$ up to $10^{21} \mathrm{eV}$ (see Fig. 4).

\section{Neutrino searches}

Neutrinos are promising astrophysical messengers, as illustrated by the several observatories designed specifically to look for high energy neutrinos, such as IceCube [32] and Antares/KM3NET [33, 34]. The astrophysical neutrinos observed by these observatories can be combined with data on UHECRs detected by the Pierre Auger Observatory. The complementarity between the observatories has been highlighted in the recent multi-messenger follow-up searches (discussed further in Sect. 4). Since they interact

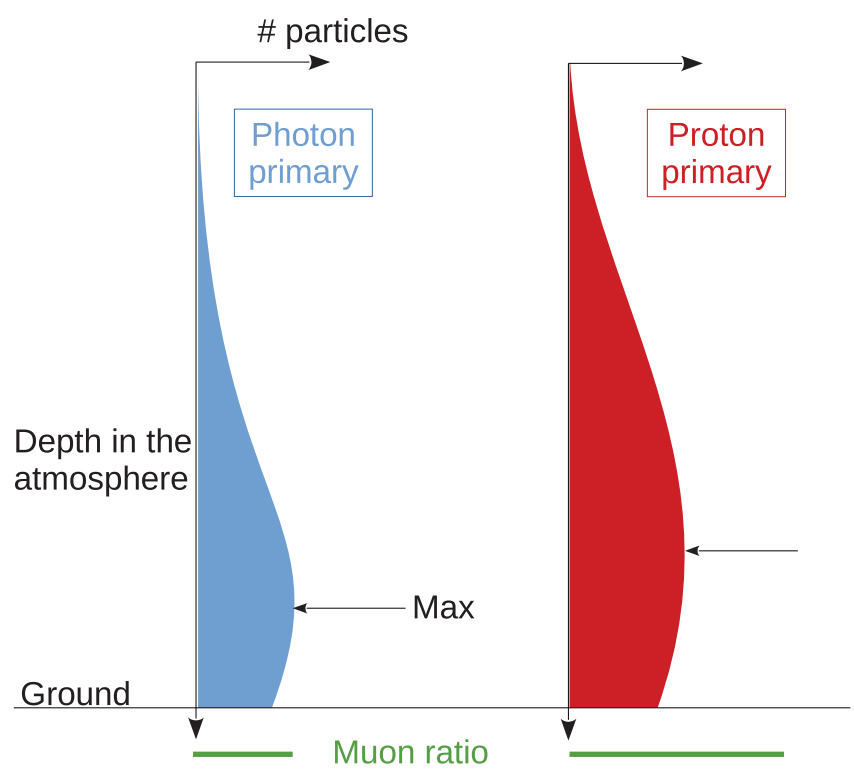

Figure 3. Scheme of the differences between photon-/protoninduced showers. EAS induced by a photon primary have a maximum development depth closer to the ground and less muons on ground compared to a proton-induced one. All quantities in this figure are simply to highlight the tendencies and should not be taken as measurements of any kind.

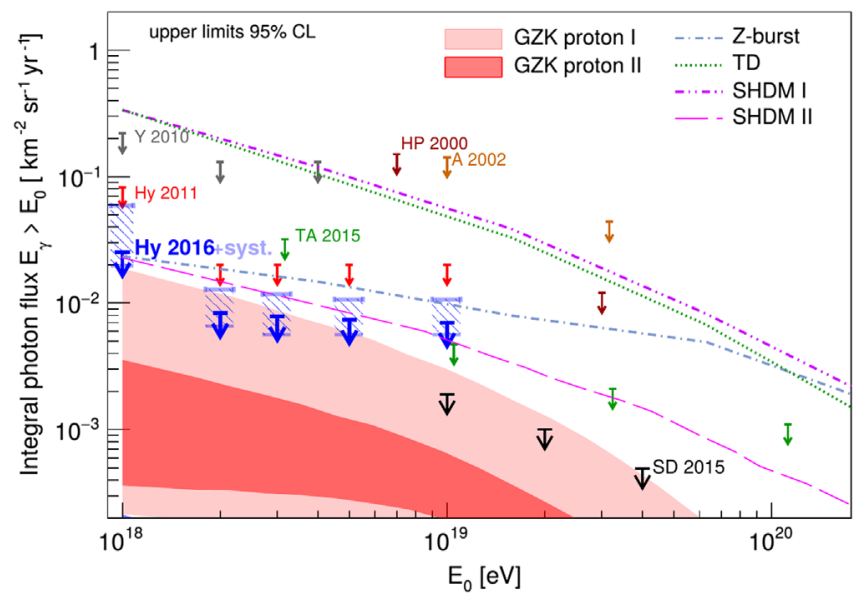

Figure 4. Upper limits on the integral photon flux for a photon flux $E^{-2}$ and no background substraction. The limits set by the Pierre Auger Observatory with hybrid data (blue arrows) and SD data (black arrows) are compared to those obtained by other experiments and predictions for GZK photon flux and top-down scenarios. Taken from [46].

only weakly with matter, neutrino-induced showers may occur very deeply in the atmosphere. One can thus search for neutrino primaries in very inclined showers since the EAS from hadronic primaries are reduced to mostly high energy muons well before reaching the SD at these high zenith angles (low background). A very inclined "young" shower (i.e., still developing when reaching the array) can only be induced by a deeply interacting neutrino. 


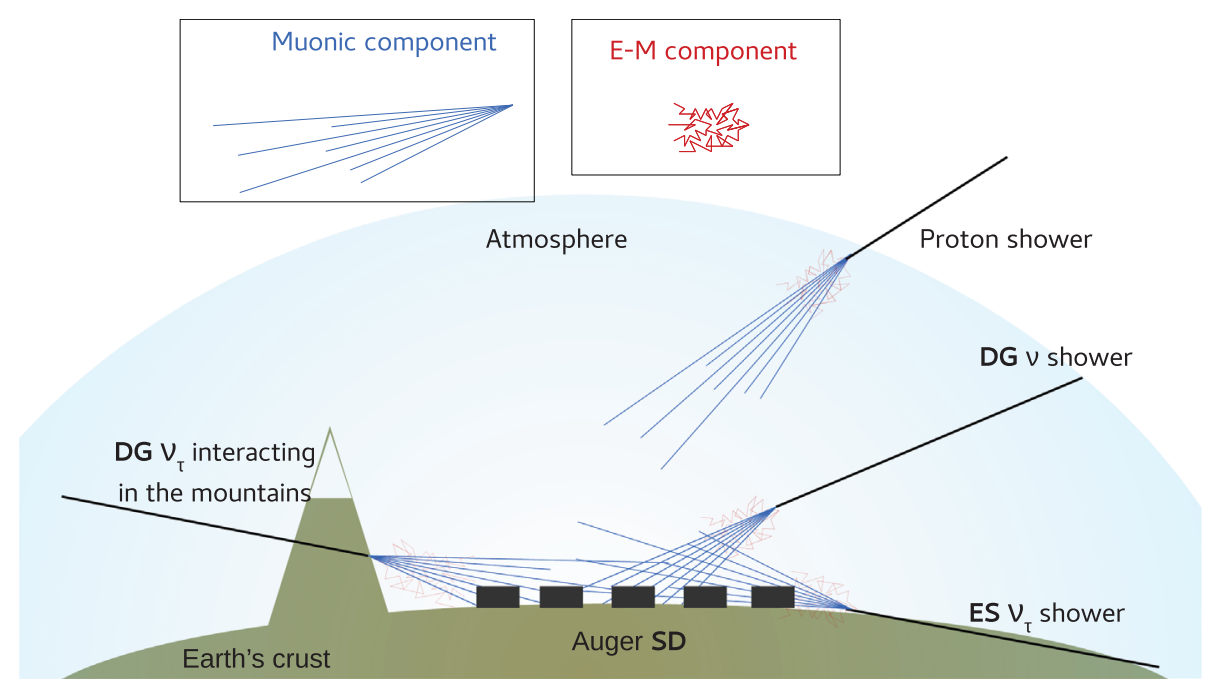

Figure 5. Scheme of the neutrino channels.

Signatures such as the time spread and the signal shapes are used to sign them with negligible hadronic background.

In Figure 5, the two channels ${ }^{2}$ that are used for neutrino searches with the Pierre Auger Observatory are illustrated: the downward going (DG) and the Earth-skimming (ES) channels [35]. The DG channel includes showers that enter the atmosphere at very high zenith angles, induced by any neutrino flavors. The DG showers are further split into two sub-channels to take into account the fact that the showers develop differently (from the point of view of the observatory) depending on their zenith angles: the downward going low $\left(60^{\circ}<\right.$ Zenith $\left.<75^{\circ}\right)$ and the downward going high $\left(75^{\circ}<\right.$ Zenith $\left.<90^{\circ}\right)$ sub-channels. The ES channel is used to look for showers initiated by tau neutrinos $\left(v_{\tau}\right)$ going through the Earth's crust. Some $v_{\tau}$ can interact close to the surface after skimming through the ground, producing a tau lepton that will start an upward-going shower above the surface array. The ES channel is looking for showers with zenith angles between $90^{\circ}$ and $95^{\circ}$. No UHE neutrinos have been identified by the Pierre Auger Observatory, although it has a very good sensitivity in the two channels [35]. This has allowed the observatory to derive limits on the diffuse flux of UHE neutrinos, comparable to the one achieved by the IceCube experiment (see Fig. 6).

\section{Follow-up searches}

Due to its good sensitivities to UHE photons and neutrinos, the Pierre Auger Observatory can play a major role in the multi-messenger astrophysics field. By combining its data with other observatories, the Pierre Auger Observatory can help pinpoint the location of potential sources, put constraints on the highest energies counterparts and broaden the energy spectrum of the observations. Prime examples of that are the large multi-messenger observations

\footnotetext{
${ }^{2}$ A channel is a range of zenith angle, between the shower axis and the ground.
}

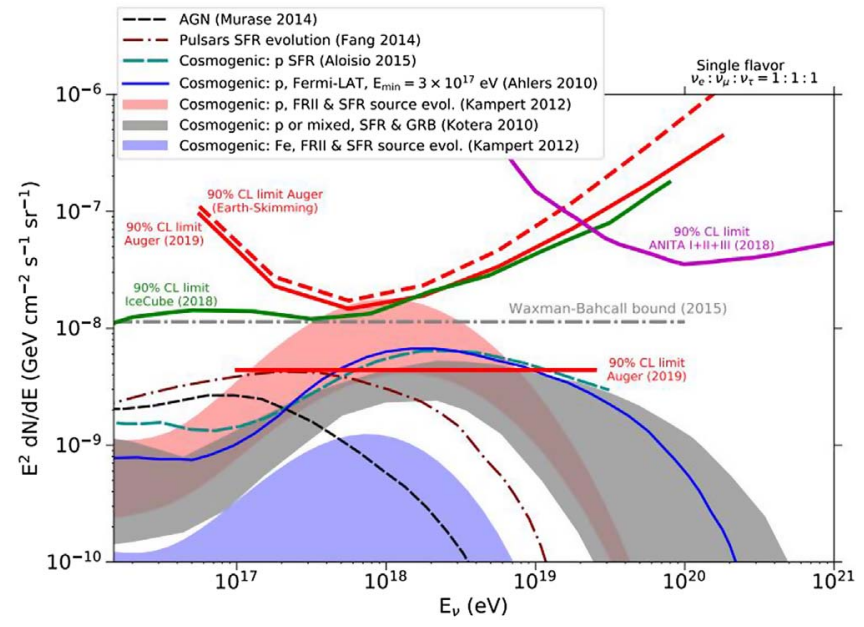

Figure 6. Upper limit to the normalization of the diffuse flux of UHE neutrino. The upper limits obtained by the Pierre Auger Observatory are compared to those obtained by IceCube and ANITA, as well as predictions from cosmogenic and astrophysical neutrino production models. Taken from [47].

campains, following-up gravitational wave events. Given the expected distance of these events from Earth $(>10 \mathrm{Mpc})$, the search for follow-up signals in the Pierre Auger Observatory data was focused on neutrino searches. More specifically, when the observatory receives an alert from another observatory about an ongoing event, a search is conducted in physically motivated time windows, around the time of the event and after it.

\section{Binary black-hole merger}

On September 14, 2015 the two Advanced LIGO observatories detected the first gravitational wave transient GW150914 [36], emitted by a binary black hole (BBH) merger. Another $\mathrm{GW}$ event from a $\mathrm{BBH}$ merger was detected on December 26, 2015 [36]. 
Table 1. Properties of the mergers reported in the GWTC-1-confident catalogue, taken from the Gravitational Wave Open Science Center database, provided by the LIGO/Virgo collaborations [49, 50].

\begin{tabular}{lccc}
\hline & GW150914 $(\mathrm{BBH})$ & GW151226 $(\mathrm{BBH})$ & GW170817 $(\mathrm{BNS})$ \\
\hline Luminosity distance $(\mathrm{Mpc})$ & $440_{-170}^{+150}$ & $450_{-190}^{+180}$ & $40_{-15}^{+7}$ \\
Primary mass $\left(M_{\odot}\right)$ & $35.6_{-3.1}^{+4.7}$ & $13.7_{-3.2}^{+8.8}$ & $1.46_{-0.10}^{+0.12}$ \\
Secondary mass $\left(M_{\odot}\right)$ & $30.6_{-4.4}^{+3.0}$ & $7.7_{-2.5}^{+2.2}$ & $1.27_{-0.09}^{+0.06}$ \\
Radiated energy $\left(M_{\odot} \times c^{2}\right)$ & $3.1_{-0.4}^{+0.4}$ & $1.0_{-0.2}^{+0.1}$ & $\geq 0.04$ \\
\hline
\end{tabular}

Those mergers are potential sources of UHECRs $[16,18,37]$ through the acceleration of matter from debris of the black holes by Fermi processes in magnetic fields. These UHECRs could then interact with the surrounding matter, producing UHE gamma-rays and neutrinos. Given that these $\mathrm{BBH}$ mergers are estimated to be located at distances $>400$ Mpc (see Tab. 1), UHE charged CRs or photons emitted from these sites would have interacted with matter or have been deviated by extragalactic magnetic fields before reaching us.

The Pierre Auger Observatory has performed a search for the remaining UHE particles, the neutrinos, to try and constrain astrophysical models. In both events, UHE neutrinos have been looked for in time-windows of $\pm 500 \mathrm{~s}$ around the estimated time of the merger and 1 day after it, corresponding to the phases of gamma ray bursts (GRBs) [38]. The $\pm 500 \mathrm{~s}$ time-window is motivated by the upper limit of the prompt phase of a GRB. The 1 day time-window after the merger corresponds to the upper limit of the afterglow phase of a GRB.

Both DG and ES channels (see Sect. 2) are used for the UHE neutrino searches. Each channel maps a different area of the sky. Figure 7 shows the fraction of the sky that was visible through the neutrino search channels of the Pierre Auger Observatory along with the expected positions of the mergers from the Fermi-GRB instrument.

The performances of the SD are constantly monitored, and they were found to be stable in the search windows.

No UHE neutrinos candidates were found in coincidence with the expected directions of the GW events. Thanks to that, the Pierre Auger collaboration was able to derive the first limits on UHE neutrinos emission in an identified GW event. The limits set on the fluence of UHE neutrinos are highly declination dependant, since the neutrinosearches are performed by analysing horizontal or upwardgoing showers (see Sect. 2). The upper bounds to the UHE neutrino spectral fluence per flavor varies between $F(E)=2(\mathrm{E} / \mathrm{GeV})^{-2} \mathrm{GeV}^{-1} \mathrm{~cm}^{-2}$ and $F(E)=$ $20(\mathrm{E} / \mathrm{GeV})^{-2} \mathrm{GeV}^{-1} \mathrm{~cm}^{-2}$ in the declination range available for neutrinos searches: $\delta \in\left[-53^{\circ}, 55^{\circ}\right]$. The most restrictive upper-limit on the total energy emmitted per flavor as neutrinos is set at declination $\delta \sim-53^{\circ}$ for GW151226:

$$
E_{v, \text { tot }}\left(\delta \sim-53^{\circ}\right)<7.7 \times 10^{53} \mathrm{erg} .
$$

The upper-limits set by IceCube/ANTARES are better: from $2.8 \mathrm{GeV} \mathrm{cm}^{-2}$ to $150 \mathrm{GeV} \mathrm{cm}{ }^{-2}$ in several energy bins at $\delta \sim-70^{\circ} \sim 5.4 \times 10^{51} \mathrm{erg}$ at declinations close to the
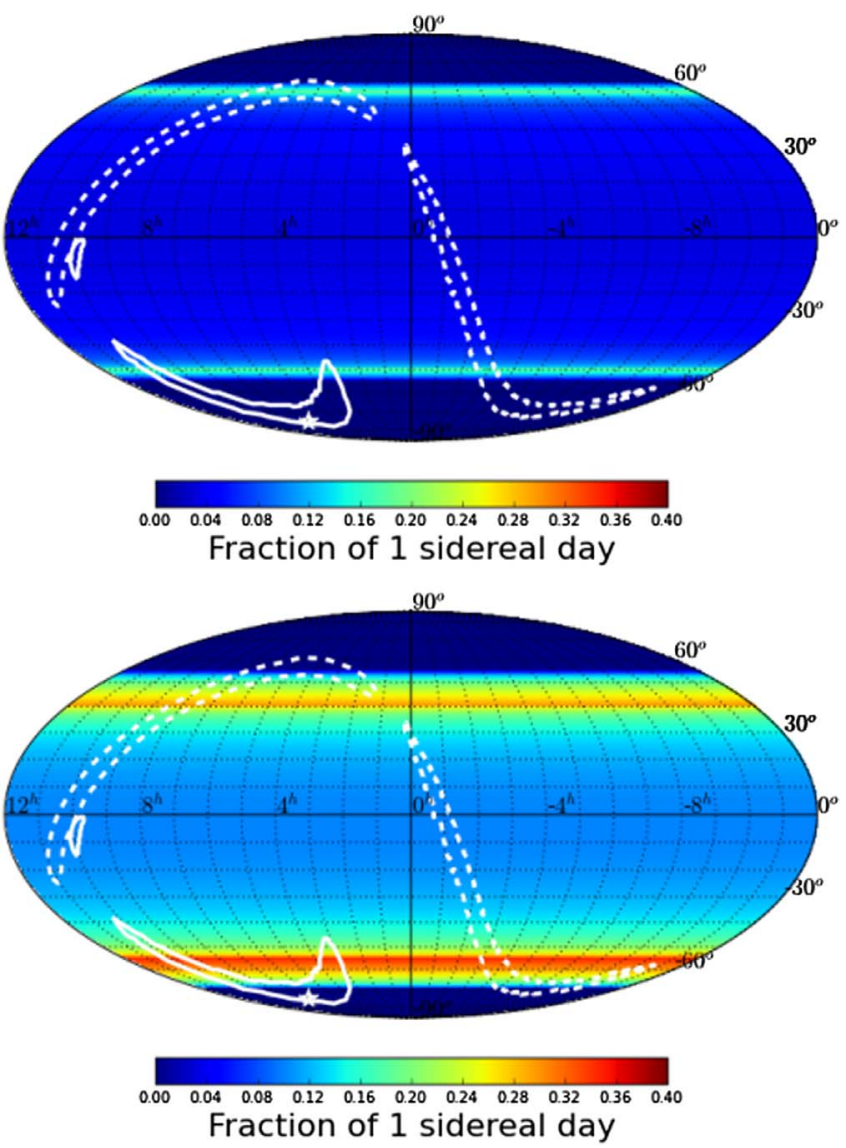

Figure 7. Skymaps in equatorial coordinates of the visibility by the Pierre Auger Observatory during both BBH events. The colorscale indicates the fraction of a day each portion of the sky is visible by the Pierre Auger Observatory SD in the ES channel (top pannel) and the DG channel (bottom pannel). The white solid line represents the $90 \%$ CL position of GW150914, the dashed line is the 90\% CL location of event GW151226 and the white star is the best-fit location of GW150914 obtained with data from the Fermi-GBM instrument. Taken from [48].

equator, but apply in the energy range $[100 \mathrm{GeV}$, $100 \mathrm{PeV}]$, while the limits from the Pierre Auger Observatory apply in the complementary range [100 PeV, $25 \mathrm{EeV}]$ [39].

\section{Binary neutron star merger}

On 2017 August 17th, the Fermi gamma-ray burst monitor detected a gamma-ray burst and sent an alert about (the later designated) GRB170817A [40]. 


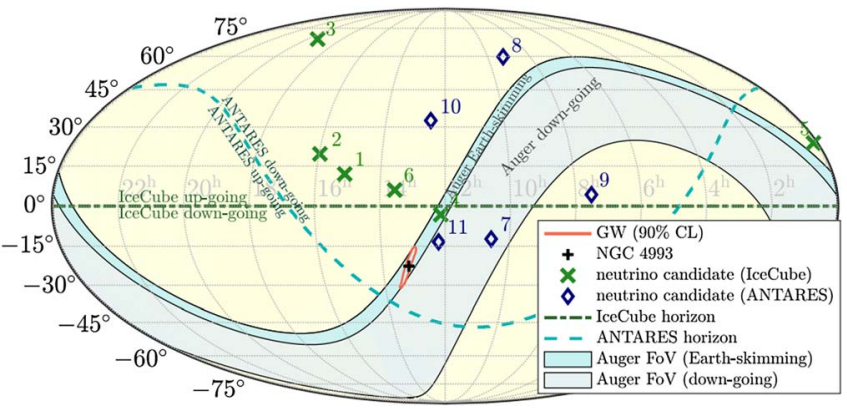

Figure 8. Skymap in equatorial coordinates during the GW170817 event. The expected localization of the BNS event and its host galaxy are shown, as well as the neutrino candidates within $\pm 500 \mathrm{~s}$ of the merger and the horizons of the ANTARES and IceCube experiments. The Pierre Auger Observatory fields of view in the ES and DG channels are also depicted [41] and references therein.

About 6 min later, the LIGO/Virgo collaboration sent its own alert, related to a GW event. This event was found inside the latency data from a single interferometer (LIGO Hanford). This was the first time an alarm was sent through a signal only seen in one interferometer but, due to its temporal proximity with the GRB alert sent by Fermi, it was judged significant enough.

Following these alerts, numerous observatories performed searches for potential counterparts to the merger, of every possible kind, be it optical, infra-red, X-rays but also neutrinos. The experiments IceCube, Antares, and the Pierre Auger Observatory looked for HE and UHE neutrinos [41].

All three observatories searched for neutrinos in timewindows of $\pm 500 \mathrm{~s}$ around the event, as well as in a 14-days time-window, to cover scenarios of longer-lived neutrino emission processes. Figure 8 is the skymap during the event, with the field-of-view (FOV) of the Pierre Auger Observatory with both ES and DG channel, the horizons and neutrino candidates of the ICeCube and Antares experiments, as well as the position of NGC 4993, the galaxy localized by optical observations as host of the merger. Remarkably, the predicted location of the merger turned out to be right in the Pierre Auger Observatory's field-ofview, in the ES channel, during the $\pm 500 \mathrm{~s}$ time-window around the event.

None of the three observatories found any neutrino candidate in the discussed search-timelines directionally correlated to the merger. This result is consistent with the expectations from an off-axis or low-luminosity GRB [41].

By combining the results from the observatories, it was possible to apply limits on the spectral fluence of emitted HE/UHE neutrinos per flavor in the two timewindows. In the $\pm 500 \mathrm{~s}$ time-winow, upper-limits from $\sim 0.5 \mathrm{GeV} \mathrm{cm}{ }^{-2}$ up to $\sim 15 \mathrm{GeV} \mathrm{cm}{ }^{-2}$ in the energy range $\left[10^{12}, 10^{20}\right] \mathrm{eV}$ have been obtained by combining observations from the three observatories. On its most sensitive energy range, from $10^{17} \mathrm{eV}$ to $2.5 \times 10^{19} \mathrm{eV}$, the upper limit set by the Pierre Auger Observatory is $F(E)=$ $0.77(\mathrm{E} / \mathrm{GeV})^{-2} \mathrm{GeV}^{-1} \mathrm{~cm}^{-2}$. In the 14 day time window, the sensitivity drops since, as the Earth rotates, the location of the source shifts in and out of the neutrino search channels. This leads to an upper-limit of the spectral fluence of $F(E)=25(\mathrm{E} / \mathrm{GeV})^{-2} \mathrm{GeV}^{-1} \mathrm{~cm}^{-2}$ over the same energy range as for the $\pm 500 \mathrm{~s}$ time-window. This search highlighted the complementarity between the three HE/UHE neutrino observatories, be it in directionality or energy range. It also opened this new era of multi-messenger and multi-temporal searches.

\section{Perspectives for the Pierre Auger Observatory}

In coming years, the Pierre Auger Observatory will continue to participate in multi-messenger searches, by looking for UHE particles in coincidence with triggering events. Improvements to the observatory are already underway, with the AugerPrime upgrade [42]. By adding new, complementary detectors, the aim of this upgrade is to achieve a better shower-to-shower identification of the primaries. Two subdetectors will be added to the SD during the upgrade: a surface scintillator detector (SSD) and a radio detector (RD). Both will consist of an array of individual detectors being added on top of the already active WCD. The SSD will also perform a detection of the secondary particles from the EAS, but with a different sensitivity to their muonic and electro-magnetic components compared to the SD. This will allow for a better dissociation between the types of secondary particles going through the surface array. The RD antennas will detect the radio signal emitted during the EAS development through the atmosphere. It is especially interesting for highly inclined showers, where the radio footprint are very long and thus are more likely to be picked up by multiple radio antennas. A procedure is being developed to perform semi-automatic follow-up searches for UHE neutrinos in the Pierre Auger Observatory data. Given that no UHE neutrino candidates have been detected so far and the low fluxes we expect, any event passing the criteria should be looked at by a human before sending any alert. However having an automated pipeline to analyse possible candidates in the context of a neutrino search allows for a quick human check and thus, if relevant, an alert could be sent quickly. In the future, projects are underway to combine the data from multiple observatories to generate alerts, like the Astrophysical Multi-Messenger Observatory Network (AMON) [43]. It would also be very interesting to combine the data from the HE/UHE neutrinos observatories to send alerts.

\section{References}

1. Linsley J (1963), Evidence for a primary cosmic-ray particle with energy $10^{20} \mathrm{eV}$. Phys Rev Lett 10, 146-148.

2. Pierre Auger Collaboration (2010), Measurement of the energy spectrum of cosmic rays above $10^{18} \mathrm{eV}$ using the Pierre Auger Observatory. Phys Lett B 685, 4-5, 239-246. 
3. Pierre Auger, Ehrenfest P, Maze R, Daudin J, Fréon Robley A (1939), Extensive cosmic ray showers. Rev. Mod. Phys. 11, 288-291.

4. Matthews J (2005), A Heitler model of extensive air showers. Astropart Phys 22, 5, 387-397.

5. Clark GW, Earl J, Kraushaar WL, Linsley J, Rossi BB, Scherb F, Scott DW (1961), Cosmic-ray air showers at sea level. Phys Rev 122, 637-654.

6. Dembinski HP, Arteaga-Velázquez JC, Cazon L, Conceição R, Gonzalez J, Itow Y, Ivanov D, Kalmykov NN, Karpikov I, Müller S, Pierog T, Riehn F, Roth M, Sako T, Soldin D, Takeishi R, Thompson G, Troitsky S, Yashin I, Zadeba E, Zhezher Y, EAS-MSU, IceCube, KASCADE-Grande, NEVOD-DECOR, Pierre Auger, SUGAR, Telescope Array, Yakutsk EAS Array collaborations (2019), Report on tests and measurements of hadronic interaction properties with air showers. EPJ Web Conf 210, 02004.

7. Abbasi RU, Abe M, Abu-Zayyad T, Allen M, Azuma R, Barcikowski E, Belz JW, Bergman DR, Blake SA, Cady R, et al. (2016), The energy spectrum of cosmic rays above $10^{17.2}$ $\mathrm{eV}$ measured by the fluorescence detectors of the Telescope Array experiment in seven years. Astropart Phys 80, 131140.

8. Chiavassa A, Apel WD, Arteaga-Velázquez JC, Bekk K, Bertaina M, Blümer J, Bozdog H, Brancus IM, Cantoni E, Cossavella F, Daumiller K, de Souza V, Di Pierro F, Doll P, Engel R, Fuhrmann D, Gherghel-Lascu A, Gils HJ, Glasstetter R, Grupen C, Haungs A, Heck D, Hörandel JR, Huber D, Huege T, Kampert K-H, Kang D, Klages HO, Link K, Łuczak P, Mathes HJ, Mayer HJ, Milke J, Mitrica B, Morello C, Oehlschläger J, Ostapchenko S, Palmieri N, Pierog T, Rebel H, Roth M, Schieler H, Schoo S, Schröder FG, Sima O, Toma G, Trinchero GC, Ulrich H, Weindl A, Wochele J, Zabierowski J (2019), Summary of the main results of the KASCADE and KASCADE-Grande experiments. EPJ Web Conf 208, 03002.

9. Yoshida S, Hayashida N, Honda K, Honda M, Imaizumi S, Inoue N, Kadota K, Kakimoto F, Kamata K, Kawaguchi S, Kawasumi N, Matsubara Y, Murakami K, Nagano M, Ohoka H, Teshima M, Tsushima I, Yoshii H (1995), The cosmic ray energy spectrum above $3 \times 10^{18} \mathrm{eV}$ measured by the Akeno Giant Air Shower Array. Astropart Phys 3, 2, 105-123.

10. Yakutsk collaboration (2004), The spectrum features of UHECRs below and surrounding GZK. Nucl Phys B Proc Suppl 136, 3-11. CRIS 2004 Proceedings of the Cosmic Ray International Seminars: GZK and Surroundings.

11. High Resolution Fly's Eye Collaboration (2008), First observation of the Greisen-Zatsepin-Kuzmin suppression. Phys Rev Lett 100, 101101.

12. Blasi P (2013), The origin of galactic cosmic rays. Astron Astrophys Rev 21, 1, 70.

13. Settimo M (2016), Review on extragalactic cosmic rays detection, in: XXV European Cosmic Ray Symposium, Turin, September 4-9, 2016.

14. Hillas AM (1984), The Origin of Ultra-High-Energy Cosmic Rays. Annu Rev Astron Astrophys 22, 425-444.

15. Alves Batista R, Biteau J, Bustamante M, Dolag K, Engel R, Ke Fang K, Karl-Heinz K, Kostunin D, Mostafa M, Murase K, Oikonomou F, Olinto AV, Panasyuk MI, Sigl G, Taylor AM, Unger M (2019), Open questions in cosmic-ray research at ultrahigh energies. Front Astron Space Sci 6, 23.

16. Kotera K, Silk J (2016), Ultrahigh Energy cosmic rays and black hole mergers. Astrophys J 823, 2, L29.

17. Semikoz D.V (2007), Constraints on top-down models for the origin of UHECRs from the Pierre Auger Observatory data, in: Proceedings, 30th International Cosmic Ray Conference
(ICRC 2007): Merida, Yucatan, Mexico, July 3-11, 2007 4, pp. $433-436$.

18. Murase K, Kashiyama K, Mészáros P, Shoemaker I, Senno N (2016), Ultrafast outflows from black hole mergers with a minidisk. Astrophys J 822, 1, L9.

19. Pierre Auger Collaboration (2015), The Pierre Auger Cosmic Ray Observatory. Nucl Instrum Methods Phys Res Sect A: Accel Spectrom Detect Assoc Equip 798, 172-213.

20. Pierre Auger Collaboration (2016), Energy estimation of cosmic rays with the engineering radio array of the Pierre Auger Observatory. Phys Rev D 93, 12, 122005.

21. Pierre Auger Collaboration (2017), Muon counting using silicon photomultipliers in the AMIGA detector of the Pierre Auger Observatory. JINST 12, 03, P03002.

22. Pierre Auger Collaboration (2008), Upper limit on the cosmic-ray photon flux above $10^{19} \mathrm{eV}$ using the surface detector of the Pierre Auger Observatory. Astropart Phys 29, 243-256.

23. Gelmini GB, Kalashev OE, Semikoz DV (2008), GZK photons as ultra high energy cosmic rays. J Exp Theor Phys 106, 1061-1082.

24. Aloisio R, Blasi P, Ghia PL, Grillo AF (2000), Probing the structure of space-time with cosmic rays. Phys Rev D 62, 053010

25. Guedes Lang R, Martínez-Huerta H, de Souza V (2018), Limits on the Lorentz invariance violation from UHECR astrophysics. Astrophys J 853, 1, 23.

26. Zatsepin GT, Kuzmin VA (1966), Upper limit of the spectrum of cosmic rays. JETP Lett 4, 78-80. [Pisma Zh. Eksp. Teor. Fiz. 4, 114 (1966)].

27. Greisen K (1966), End to the cosmic-ray spectrum? Phys Rev Lett 16, 748-750.

28. Pierre Auger Collaboration (2017), Combined fit of spectrum and composition data as measured by the Pierre Auger Observatory. JCAP 1704, 04, 038. [Erratum: JCAP 1803, no. 03, E02 (2018)].

29. Cazon L (2019), Probing high-energy hadronic interactions with extensive air showers. PoS ICRC2019, 005.

30. Domenico M De, Settimo M, Riggi S, Bertin E (2013), Reinterpreting the development of extensive air showers initiated by nuclei and photons. JCAP 1307, 050.

31. Bleve C (2016), Updates on the neutrino and photon limits from the Pierre Auger Observatory. PoS ICRC2015, 1103.

32. Collaboration IceCube (2017), The IceCube Neutrino Observatory: instrumentation and online systems. JINST 12, 03, P03012.

33. ANTARES Collaboration (2011), ANTARES: the first undersea neutrino telescope. Nucl Instrum Methods Phys Res A 656, 1, 11-38.

34. Aiello S, Akrame SE, Ameli F, Anassontzis EG, Andre M, Androulakis G, Anghinolfi M, Anton G, Ardid M, Aublin J, Avgitas T, Bagatelas C, Barbarino G, Baret B, Barrios-Martí J, Belias A, Berbee E, Van Den Berg A, Bertin V, Biagi S, Biagioni A, Biernoth C, Boumaaza J, Bourret S, Bouta M, Bouwhuis M, Bozza C, Brânzaş H, Bruchner M, Bruijn R, Brunner J, Buis E, Buompane R, Busto J, Calvo D, Capone A, Celli S, Chabab M, Chau N, Cherubini S, Chiarella V, Chiarusi T, Circella M, Cocimano R, Coelho JAB, Coleiro A, Colomer Molla M, Coniglione R, Coyle P, Creusot A, Cuttone G, D'Onofrio A, Dallier R, Distefano C, Domi A, Donà R, Donzaud C, Dornic D, Dörr M, Durocher M, Eberl T, Van Eijk D, El Bojaddaini I, Eljarrari H, Elsaesser D, Enzenhöfer A, Fermani P, Ferrara G, Filipović MD, Fusco LA, Gal T, Garcia A, Garufi F, Gialanella L, Giorgio E, Giuliante A, Gozzini SR, Gracia R, Graf K, Grasso D, Grégoire T, Grella G, Hallmann S, Hamdaoui H, Van Haren H, 
Heid T, Heijboer A, Hekalo A, Hernández-Rey JJ, Hofestädt J, Illuminati G, James CW, Jongen M, De Jong M, De Jong P, Kadler M, Kalaczyński P, Kalekin O, Katz UF, Khan Chowdhury NR, Kießling D, Koffeman EN, Kooijman P, Kouchner A, Kreter M, Kulikovskiy V, Kunhikannan Kannichankandy M, Lahmann R, Larosa G, Le Breton R, Leone F, Leonora E, Levi G, Lincetto M, Lonardo A, Longhitano F, Lopez Coto D, Lotze M, Maderer L, Maggi G, Mańczak J, Mannheim K, Margiotta A, Marinelli A, Markou C, Martin L, Martínez-Mora JA, Martini A, Marzaioli F, Mele R, Melis KW, Migliozzi P, Migneco E, Mijakowski P, Miranda LS, Mollo CM, Morganti M, Moser M, Moussa A, Muller R, Musumeci M, Nauta L, Navas S, Nicolau CA, Nielsen C, Ó Fearraigh B, Organokov M, Orlando A, Ottonello S, Panagopoulos V, Papalashvili G, Papaleo R, Păvălas GE, Pellegrino C, Perrin-Terrin M, Piattelli P, Pikounis K, Pisanti O, Poirè C, Polydefki G, Popa V, Post M, Pradier T, Pühlhofer G, Pulvirenti S, Quinn L, Raffaelli F, Randazzo N, Razzaque S, Real D, Resvanis L, Reubelt J, Riccobene G, Richer M, Rigalleau L, Rovelli A, Saffer M, Salvadori I, Samtleben DFE, Sánchez Losa A, Sanguineti M, Santangelo A, Santonocito D, Sapienza P, Schumann J, Sciacca V, Seneca J, Sgura I, Shanidze R, Sharma A, Simeone F, Sinopoulou A, Spisso B, Spurio M, Stavropoulos D, Steijger J, Stellacci SM, Strandberg B, Stransky D, Stüven T, Taiuti M, Tatone F, Tayalati Y, Tenllado E, Thakore T, Trovato A, Tzamariudaki E, Tzanetatos D, Van Elewyck V, Versari F, Viola S, Vivolo D, Wilms J, De Wolf E, Zaborov D, Zornoza JD, Zúñiga J (2019), Neutrino telescope to point-like neutrino sources. Astropart. Phys. 111, 100-110.

35. Pierre Auger Collaboration (2015), Improved limit to the diffuse flux of ultrahigh energy neutrinos from the Pierre Auger Observatory. Phys Rev D 91, 9, 092008.

36. LIGO Scientific Collaboration and Virgo Collaboration (2016), Binary black hole mergers in the first advanced LIGO observing run. Phys Rev X 6, 041015.

37. Biermann PL, Caramete LI, Fraschetti F, Gergely LA, Harms BC, Kun E, Lundquist JP, Meli A, Nath BB, Seo E-S, Stanev T, Tjus JB (2017), The nature and origin of ultrahigh energy cosmic ray particles. Frascati Phys Ser 64, 103121.

38. Mészáros P (2006), Gamma-ray bursts. Rep Prog Phys 69, 8, 2259-2321.

39. ANTARES Collaboration, IceCube Collaboration, LIGO Scientific Collaboration, Virgo Collaboration (2016),
High-energy neutrino follow-up search of gravitational wave event GW150914 with ANTARES and IceCube. Phys Rev D 93, 12,122010 .

40. BOOTES Collaboration, MWA, The CALET Collaboration, IKI-GW Follow-up Collaboration, H.E.S.S. Collaboration, LOFAR Collaboration, LWA, HAWC Collaboration, The Pierre Auger Collaboration, ALMA Collaboration, Euro VLBI Team, Pi of the Sky Collaboration, The Chandra Team at McGill University, DFN, ATLAS, High Time Resolution Universe Survey, RIMAS, RATIR, SKA South Africa/MeerKAT (2017), Multi-messenger observations of a binary neutron star merger. Astrophys J 848, 2, L12.

41. ANTARES Collaboration, IceCube Collaboration, Pierre Auger Collaboration (2017), Search for high-energy neutrinos from binary neutron star merger GW170817 with ANTARES, IceCube, and the Pierre Auger Observatory. Astrophys J 850, 2, L35.

42. Castellina A (2019), AugerPrime: the Pierre Auger Observatory upgrade. EPJ Web Conf 210, 06002.

43. Ayala Solares HA, Coutu S, Cowen DF, Delaunay JJ, Fox DB, Keivani A, Mostafá M, Murase K, Oikonomou F, SeglarArroyo M, et al. (2020), The astrophysical multimessenger observatory network (AMON): performance and science program. Astropart Phys 114, 68-76.

44. Particle Data Group (2018), Review of particle physics. Phys Rev D 98, 3, 030001.

45. Beatty J, Westerhoff S (2009), The highest-energy cosmic rays. Annu Rev Nucl Part Sci 59, 319-345.

46. Pierre Auger Collaboration (2017), Search for photons with energies above $10^{18} \mathrm{eV}$ using the hybrid detector of the Pierre Auger Observatory. JCAP 1704, 04, 009.

47. Pierre Auger Collaboration (2019), Probing the origin of ultra-high-energy cosmic rays with neutrinos in the EeV energy range using the Pierre Auger Observatory. JCAP 10, 10,022 .

48. Pierre Auger Collaboration (2016), Ultrahigh-energy neutrino follow-up of gravitational wave events GW150914 and GW151226 with the Pierre Auger Observatory. Phys Rev D 94, 12, 122007.

49. LIGO Scientific Collaboration and Virgo Collaboration (2019), GWTC-1: a gravitational-wave transient catalog of compact binary mergers observed by LIGO and Virgo during the first and second observing runs. Phys Rev X9, 3, 031040.

50. LIGO Scientific Collaboration, Virgo Collaboration, Abbott R, et al. Gravitationnal Wave Open Science Center. Arxiv eprint: arXiv:1912.11716

Cite this article as: Souchard J 2020. Multi-messengers at ultra-high energies with the Pierre Auger Observatory. 4open, 3, 4. 